\title{
Laparoscopic versus open left hemihepatectomy for hepatocellular carcinoma: a propensity score matching analysis
}

\author{
Yong Yi ${ }^{1,2 \#}$, Jialei Weng ${ }^{1,2 \#}$, Chenhao Zhou ${ }^{1,2 \#}$, Gao Liu ${ }^{1,2}$, Ning $\operatorname{Ren}^{1,2,3}$ \\ ${ }^{1}$ Department of Liver Surgery and Transplantation, Liver Cancer Institute, Zhongshan Hospital, Fudan University, Shanghai, China; ${ }^{2}$ Key \\ Laboratory of Carcinogenesis and Cancer Invasion of Ministry of Education, Shanghai, China; ${ }^{3}$ Institute of Fudan Minhang Academic Health \\ System, and Key Laboratory of Whole-period Monitoring and Precise Intervention of Digestive Cancer (SMHC), Minhang Hospital \& AHS, Fudan \\ University, Shanghai, China \\ Contributions: (I) Conception and design: N Ren; Y Yi; (II) Administrative support: N Ren; (III) Provision of study materials or patients: Y Yi; C \\ Zhou; (IV) Collection and assembly of data: J Weng; G Liu; (V) Data analysis and interpretation: J Weng; C Zhou; (VI) Manuscript writing: All \\ authors; (VII) Final approval of manuscript: All authors. \\ \#These authors contributed equally to this work. \\ Correspondence to: Ning Ren. Department of Liver Surgery and Transplantation, Liver Cancer Institute, Zhongshan Hospital, Fudan University, 180 \\ Fenglin Road, Xuhui District, Shanghai 200032, China. Email: ren.ning@zs-hospital.sh.cn.
}

Background: Despite the increasing application of laparoscopic hepatectomy (LH) for hepatocellular carcinoma (HCC), laparoscopic left hemihepatectomy (LLH) remains a relatively rare surgery and comparison of perioperative and oncological outcomes between LLH and open left hemihepatectomy (OLH) is lacking.

Methods: A total of 276 HCC patients who underwent either LLH or OLH between January 2008 and November 2019 were enrolled in this retrospective observational study and a 1:2 propensity score matching (PSM) was performed between LLH and OLH groups.

Results: Patients in LLH group had smaller tumor size $(\mathrm{P}=0.001)$ and earlier TNM staging $(\mathrm{P}=0.022)$ before matching. Despite the similar transfusion rate after matching, patients undergoing LLH $(\mathrm{n}=27)$ experienced less intraoperative blood loss (100.0 versus $200.0 \mathrm{~mL} ; \mathrm{P}=0.034)$ and application of hepatic portal occlusion ( $11.1 \%$ versus $63.5 \%$; $\mathrm{P}<0.001)$ compared to those in OLH group $(\mathrm{n}=52)$. There were no statistical differences of resection margin and incidence of complications between the groups. The overall survival (OS) and disease-free survival (DFS) of patients in LLH group were comparable to OLH group (3-year OS rate: 75.0\% versus $84.9 \%$; 1 -year DFS rate: $92.3 \%$ versus $92.2 \%)$.

Conclusions: LLH may be a feasible and safe alternative to OLH for selected HCC cases, providing potential short-term benefits without compromising oncologic adequacy and prognosis.

Keywords: Laparoscopic left hemihepatectomy (LLH); hepatocellular carcinoma (HCC); perioperative outcomes

Submitted Mar 18, 2020. Accepted for publication Aug 07, 2020.

doi: $10.21037 /$ tcr-20-1573

View this article at: http://dx.doi.org/10.21037/tcr-20-1573

\section{Introduction}

With the development of instruments, innovation of technology and accumulation of experience, past decades have seen an evolution of laparoscopic hepatectomy ( $\mathrm{LH})$ in hepatocellular carcinoma (HCC) treatment from local resection towards segmentectomy and even hemihepatectomy.
Furthermore, increasing evidence has indicated the technical feasibility and operation safety of LH in HCC, as well as better perioperative benefits in comparison with conventional laparotomy (1-10). Although there is a trend of LH to replace open liver resection in some experienced institutes (11), the long-term survival benefit of $\mathrm{LH}$ is lack of sufficient supports and thus more follow-up studies and randomized controlled 
trials (RCT) are warranted.

HCC is the sixth most common cancer and the fourth leading cause of cancer death worldwide (12). Liver resection is considered as one of the most effective treatments for HCC $(13,14)$. With the development of minimally invasive surgery, laparoscopic left lateral segmentectomy has also been regarded as the standard procedure and performed routinely in HCC (15). However, laparoscopic hemihepatectomy remains a relatively rare surgery because of rigorous technical demands, complicated operational procedures and potentially huge risk. In this case, there are only a few studies reporting the superior outcomes like less intraoperative blood loss and postoperative complications of laparoscopic hemihepatectomy compared to open approach (16-18). However, these studies, with a small cohort of patients recruited, either focus on right hepatectomy or fail to adopt matching design. Given that major hepatectomy is an optimal choice for HCC patients with large tumors or better liver function reserve (18), it is imperative to further authenticate the feasibility, safety and real survival benefits of laparoscopic hemihepatectomy, especially left hepatectomy, in HCC.

Hence, in this study, we aimed to compare the perioperative and long-term outcomes of laparoscopic left hepatectomy (LLH) and open left hepatectomy (OLH) in HCC. We present the following article in accordance with the STROBE reporting checklist (available at http://dx.doi. org/10.21037/tcr-20-1573).

\section{Methods}

\section{Patients and data}

This is a retrospective observational study with a matching analysis. A total of 276 patients who received left hemihepatectomy for HCC with either a laparoscopic or an open approach at Zhongshan Hospital of Fudan University, Shanghai, China between January 2008 and November 2019 were included. The inclusion and exclusion criteria of patients were as following: (I) All patients underwent primary curative LLH or OLH; (II) clinical diagnosis of HCC was confirmed by postoperative pathology; (III) no history of other malignancy; (IV) with Child-Pugh class A or early B liver function; (V) no evidence of extrahepatic metastasis or portal vein or inferior vena cava cancer embolus; (VI) open conversion cases were excluded; (VII) emergency operation due to tumor rupture was also excluded. LLH and OLH were performed by the same team of professional liver surgeons and selection of surgical approach was surgeon dependent. Among these patients, 35 individuals underwent LLH and other 241 patients were operated with OLH. The study was conducted in accordance with the Declaration of Helsinki (as revised in 2013). A written informed consent for operation was obtained from each patient and this study was approved by the Clinical Research Ethics Committee of Zhongshan Hospital, Fudan University (ID: Y2018-115).

Demographic characteristics, preoperative imaging and laboratory, operative record, and pathologic report of all patients were collected from electrical medical record system. Postoperative complications were graded according to the Clavien-Dindo classification (19). Postoperative follow-up, mainly encompassing abdominal ultrasound and serological tumor biomarkers, was conducted every 3 months. Recurrence was confirmed by enhanced computed tomography (CT) or magnetic resonance imaging (MRI). The interval between the time of surgery and the date of either death or the last follow-up time was considered as overall survival (OS). Disease-free survival (DFS) was defined as the time from the date of operation to the point of the recurrence during the follow-up period. For patients without recurrence, DFS was censored on the date of death or the last follow-up time.

\section{Propensity score matching (PSM)}

To minimize selection bias in this nonrandomized observational study, PSM analysis was employed to balance the distribution of covariates between two groups (20). The following variables were considered seriously and finally enrolled into PSM model: age, gender, alpha-fetoprotein (AFP) level, Tumor Node Metastasis (TNM) staging, presence of cirrhosis or ascites, tumor size and differentiation, and micro-vascular invasion (MVI). A propensity score of each variable was calculated by logistic regression and a 1:2 ratio matching between LLH and OLH groups was achieved by nearest neighbor method. PSM analysis was performed by the "MatchIt" package of $\mathrm{R}$ software (version 3.6.1).

\section{Surgical procedure}

In an LLH, the patient lay in the French position under general anesthesia. Pneumoperitoneum was established after the insertion of a $12-\mathrm{mm}$ port into a supraumbilical cutdown and was maintained at around $15 \mathrm{mmHg}$. Generally, four working ports were applied as follows: three 
5 -mm ports in the right upper, left upper and right flank quadrants and a $10-\mathrm{mm}$ port in the left flank quadrant.

First, the hepatic artery and the portal vein were well exposed by meticulous blunt dissection. The left branches of these two main vessels were then clamped with absorbable clips to achieve selective left inflow occlusion. Left hepatic parenchymal transection was performed using a Harmonic scalpel and hemostasis was conducted by hem-o-lock clips and bipolar electrocoagulation. Finally, after ligation of left hepatic vein, the specimen was put into a plastic bag and retrieved through an extended abdominal incision.

In an OLH, the patient was placed in the supine position and a subcostal incision was performed routinely. The procedures of surgery were similar to LLH. Briefly, parenchymal transection was performed after occlusion of left hepatic artery and left portal vein. Fibrin glue was also applied to ensure the hemostasis. An abdominal drainage tube was placed after LLH or OLH.

\section{Statistical analysis}

Data was expressed as mean \pm standard deviation (SD), median with range or count with percentage. Statistical analyses were performed by SPSS software (22.0; IBM, Armonk, NY, USA). Student's $t$-test or Mann-Whitney U test was chosen based on the data distribution for comparison of continuous variables. Pearson Chi-square test and Fisher's exact test were employed to compare discrete variables. The survival curves of OS and DFS were plotted using KaplanMeier method and difference of survival between LLH and OLH group was tested by log-rank test. A two-tailed $\mathrm{P}$ value $<0.05$ was considered as statistical significance.

\section{Results}

\section{Baseline and pathologic characteristics}

A total of 276 patients were enrolled in the study with 35 individuals in LLH group and 241 in OLH group. As listed in Table 1, there were significant differences of several baseline characteristics between the two groups such as previous abdominal surgery $(\mathrm{P}=0.043)$, albumin $(\mathrm{P}<0.001)$, prothrombin time $(\mathrm{PT})(\mathrm{P}=0.024)$ and $\mathrm{AFP}(\mathrm{P}=0.009)$. Other demographic variables, like age, gender, comorbidity, hepatitis, other laboratory indexes, hepatitis B virus (HBV), and ascites did not meet the statistical significance. PSM analysis was then performed and well-balanced baseline characteristics except albumin $(\mathrm{P}=0.015)$ between LLH $(\mathrm{n}=27)$ and OLH $(\mathrm{n}=52)$ groups were observed after matching. Similarly, LLH and OLH groups differed before $P S M$ in terms of tumor size $(\mathrm{P}=0.001)$, cell differentiation $(\mathrm{P}=0.020)$, MVI $(\mathrm{P}=0.001)$, and TNM staging $(\mathrm{P}=0.022)$ (Table 2). Subsequent matching successfully eliminated the statistical difference of these pathological features between the two groups. No significant difference was found in tumor number, tumor border, tumor encapsulation, tumor necrosis and hemorrhage, and liver cirrhosis before and after PSM.

\section{Difference of surgical outcomes between LLH and OLH groups after PSM}

We further retrospectively compared the surgery details between LLH and OLH groups after PSM. As shown in Table 3, the median intraoperative blood loss was significantly less in LLH group compared to OLH group (100.0 versus $200.0 \mathrm{~mL} ; \mathrm{P}=0.034$ ). There was no significant difference between the two groups regarding the transfusion rate $(0.0 \%$ versus $3.8 \% ; \mathrm{P}=0.544)$. In addition, the rate of hepatic portal occlusion in LLH group was significantly lower than OLH group $(11.1 \%$ versus $63.5 \%$; $\mathrm{P}<0.001)$. Resection margin, determined by pathology, was defined as the closest distance of surgical margin to tumor. It showed that the mean resection margin was comparable between the groups ( 1.2 versus $1.0 \mathrm{~cm} ; \mathrm{P}=0.597)$. None of the patients in LLH group developed complications greater than ClavienDindo classification II, while there was atrial fibrillation $(\mathrm{n}=1)$, infection $(\mathrm{n}=2)$, delirium $(\mathrm{n}=1)$, jaundice $(\mathrm{n}=1)$, and the need for blood transfusion $(\mathrm{n}=1)$ or thoracentesis $(\mathrm{n}=1)$ in OLH group $(\mathrm{P}=0.116)$.

\section{Difference of long-term survival between LLH and OLH groups after PSM}

The median follow-up was 12.40 months (range, 1.43 to 46.40 months) in LLH group and 32.78 months (range, 9.77 to 128.80 months) in OLH group. HCC recurrences were observed in four patients undergoing LLH and 12 patients in OLH group. One patient $(3.70 \%)$ in LLH group and three patients $(5.77 \%)$ in OLH group succumbed to tumor recurrence or metastasis. The OS and DFS of patients receiving LLH were comparable to those in OLH group $(\mathrm{P}=0.8401 ; \mathrm{P}=0.3655$, respectively) (Figure 1).

\section{Discussion}

Since the first report of laparoscopic liver resection by 
Table 1 Comparison of baseline characteristics between LLH and OLH groups before and after PSM

\begin{tabular}{|c|c|c|c|c|c|c|}
\hline Characteristics & \multicolumn{3}{|c|}{ Before matching } & \multicolumn{3}{|c|}{ After matching } \\
\hline Age (years) & $56.3 \pm 14.2$ & $56.2 \pm 11.8$ & $0.967^{\star}$ & $58.0 \pm 12.1$ & $57.4 \pm 10.8$ & $0.824^{\star}$ \\
\hline Gender, n (\%) & & & 0.436 & & & 0.366 \\
\hline Male & $27(77.1)$ & $199(82.6)$ & & $20(74.1)$ & $43(82.7)$ & \\
\hline Comorbidity, n (\%) & & & $0.281^{\dagger}$ & & & 0.146 \\
\hline Hypertension & $9(25.7)$ & $42(17.4)$ & & $6(22.2)$ & $9(17.3)$ & \\
\hline Diabetes & $6(17.1)$ & $18(7.5)$ & & $5(18.5)$ & $3(5.8)$ & \\
\hline CVA & $0(0.0)$ & $6(2.5)$ & & $0(0.0)$ & $3(5.8)$ & \\
\hline PAS, n (\%) & $10(28.6)$ & $36(14.9)$ & 0.043 & 7 (25.9) & $6(11.5)$ & 0.188 \\
\hline \multicolumn{7}{|l|}{ Laboratory index } \\
\hline TB $(\mu \mathrm{mol} / \mathrm{L})$ & $11.5(5.2-34.7)$ & $11.2(3.8-192.0)$ & 0.684 & $11.5(5.2-29.9)$ & $10.2(4.9-38.3)$ & 0.249 \\
\hline $\mathrm{DB}(\mu \mathrm{mol} / \mathrm{L})$ & $4.0(1.1-8.6)$ & $4.4(0.2-90.1)$ & 0.302 & $4.0(1.1-8.6)$ & $4.4(1.9-16.4)$ & 0.377 \\
\hline Albumin (g/L) & $44.0(34.0-58.0)$ & $41.0(21.0-50.0)$ & $<0.001$ & $44.0(34.0-49.0)$ & $41.0(30.0-50.0)$ & 0.015 \\
\hline ALT (U/L) & $27.0(8.0-86.0)$ & $31.0(6.0-625.0)$ & 0.303 & $29.0(8.0-86.0)$ & $29.0(9.0-625.0)$ & 0.934 \\
\hline AST (U/L) & $27.0(16.0-50.0)$ & $31.0(12.0-656.0)$ & 0.152 & $27.0(16.0-50.0)$ & $28.5(15.0-656.0)$ & 0.860 \\
\hline $\mathrm{Hb}(\mathrm{g} / \mathrm{L})$ & $145.5 \pm 16.3$ & $141.0 \pm 15.3$ & $0.105^{\star}$ & $147.4 \pm 14.9$ & $140.7 \pm 16.6$ & $0.086^{\star}$ \\
\hline Ascites, n (\%) & $0(0.0)$ & $7(2.9)$ & $0.601 \dagger$ & $0(0.0)$ & $2(3.8)$ & $0.544^{\dagger}$ \\
\hline
\end{tabular}

*, Student's $t$-test; ${ }^{\dagger}$, Fisher's exact test. AFP, indicates alpha-fetoprotein; ALT, alanine transaminase; AST, aspartate transaminase; CD, coronary disease; CVA, cerebrovascular accident; DB, direct bilirubin; Hb, hemoglobin; HBV, hepatitis B virus; LLH, laparoscopic left hemihepatectomy; OLH, open left hemihepatectomy; PAS, previous abdominal surgery; PSM, propensity score matching; PT, prothrombin time; TB, total bilirubin; WBC, white blood cells.

Reich et al. in 1991 (21), past decades have witnessed the increasing application of laparoscopic surgery in liver disease, including HCC. After the course of technique innovation and procedure exploration in the first 10 years of 21 st century, recent attentions have been focused on evaluation of the performance of laparoscopic liver resection over conventional laparotomy (11). Several advantages of laparoscopic minor hepatic resection, sectionectomy, and segmentectomy over open approach, like less intraoperative blood loss (1,5,22-26), shorter postoperative hospital stay $(1,5,22-28)$, have been widely reported by surgeons. Nevertheless, there is no strong evidence like RCT supporting these conclusions and replacement of open liver resection by $\mathrm{LH}$ is still challenging.

An important challenge is to achieve anatomical resection of HCC through laparoscopy. Anatomic resection, such 
Table 2 Comparison of pathologic characteristics between LLH and OLH groups before and after PSM

\begin{tabular}{|c|c|c|c|c|c|c|}
\hline Characteristics & \multicolumn{3}{|c|}{ Before matching } & \multicolumn{3}{|c|}{ After matching } \\
\hline Tumor size $(\mathrm{cm})$ & $5.2 \pm 2.2$ & $6.7 \pm 3.8$ & $0.001^{*}$ & $5.1 \pm 2.3$ & $5.3 \pm 2.9$ & $0.785^{\star}$ \\
\hline Tumor number, n (\%) & & & 1.000 & & & $0.114^{\dagger}$ \\
\hline Solitary & $33(94.3)$ & 224 (92.9) & & $25(92.6)$ & $52(100.0)$ & \\
\hline Tumor border, n (\%) & & & 0.155 & & & 0.514 \\
\hline Clear & $35(100.0)$ & $221(91.7)$ & & 27 (100.0) & $49(94.2)$ & \\
\hline Unclear & $0(0.0)$ & $20(8.3)$ & & $0(0.0)$ & $3(5.8)$ & \\
\hline Encapsulation, n (\%) & & & 0.115 & & & $0.246^{\dagger}$ \\
\hline None & $5(14.3)$ & $75(31.1)$ & & $3(11.1)$ & $14(26.9)$ & \\
\hline Differentiation, n (\%) & & & $0.020^{\dagger}$ & & & $0.659^{\dagger}$ \\
\hline Grade I & $1(2.9)$ & $9(3.7)$ & & $1(3.7)$ & $4(7.7)$ & \\
\hline Grade II & $21(60.0)$ & $194(80.5)$ & & $19(70.4)$ & $38(73.1)$ & \\
\hline Grade III & $13(37.1)$ & $37(15.4)$ & & 7 (25.9) & $10(19.2)$ & \\
\hline Grade IV & $0(0.0)$ & $1(0.4)$ & & $0(0.0)$ & $0(0.0)$ & \\
\hline Tumor necrosis, n (\%) & $8(22.9)$ & $61(25.3)$ & 0.754 & $5(18.5)$ & $13(25.0)$ & 0.515 \\
\hline Tumor hemorrhage, n (\%) & $1(2.9)$ & $2(0.8)$ & $0.335^{\dagger}$ & $1(3.7)$ & $0(0.0)$ & $0.342^{\dagger}$ \\
\hline IIIB & $0(0.0)$ & $0(0.0)$ & & $0(0.0)$ & $0(0.0)$ & \\
\hline IIIC & $0(0.0)$ & $7(2.9)$ & & $0(0.0)$ & $0(0.0)$ & \\
\hline Cirrhosis, n (\%) & $24(68.6)$ & $159(66.0)$ & 0.761 & $18(66.7)$ & $34(65.4)$ & 0.909 \\
\hline
\end{tabular}

*, Student's $t$-test; ${ }^{\dagger}$, Fisher's exact test. LLH, indicates laparoscopic left hemihepatectomy; OLH, open left hemihepatectomy; PSM, propensity score matching; TNM, Tumor Node Metastasis.

as major hepatectomy and hemihepatectomy, is preferred considering the malignancy of the disease and the intention of curative resection (29). Some studies have indicated that laparoscopic anatomic hepatectomy is feasible in selected patients with the premise of rich experience, committed practice and proper coaching $(30,31)$. However, this technology is limited to a few advanced medical centers because of the technically demanding procedure and thus relevant studies are lacking.

Here, this study enrolled 276 patients who received left hemihepatectomy for HCC in our hospital. We noticed that some patients undergoing LLH had cirrhosis or a tumor size larger than $5 \mathrm{~cm}$. Cirrhosis can increases the risk of intraoperative bleeding and postoperative liver failure (32). 
Table 3 Comparison of surgical outcomes between LLH and OLH groups after PSM

\begin{tabular}{lccc}
\hline Outcomes & LLH $(\mathrm{n}=27)$ & OLH $(\mathrm{n}=52)$ & $\mathrm{P}$ \\
\hline Blood loss $(\mathrm{mL})$ & $100.0(10.0-800.0)$ & $200.0(10.0-1,000.0)$ & 0.034 \\
Blood transfusion, $\mathrm{n}(\%)$ & $0(0.0)$ & $2(3.8)$ & $0.544^{\dagger}$ \\
Hepatic portal occlusion & $3(11.1)$ & $33(63.5)$ & $<0.001$ \\
Resection margin (cm) & $1.2 \pm 1.0$ & $1.0 \pm 0.7$ & 0.597 \\
Clavien-Dindo grade, $\mathrm{n}(\%)$ & & & $0.116^{\dagger}$ \\
I & $27(100.0)$ & $45(86.6)$ & $6(11.5)$ \\
II & $0(0.0)$ & $1(1.9)$ & \\
Illa & $0(0.0)$ &
\end{tabular}

${ }^{\dagger}$, Fisher's exact test. LLH, indicates laparoscopic left hemihepatectomy; OLH, open left hemihepatectomy; PSM, propensity score matching.
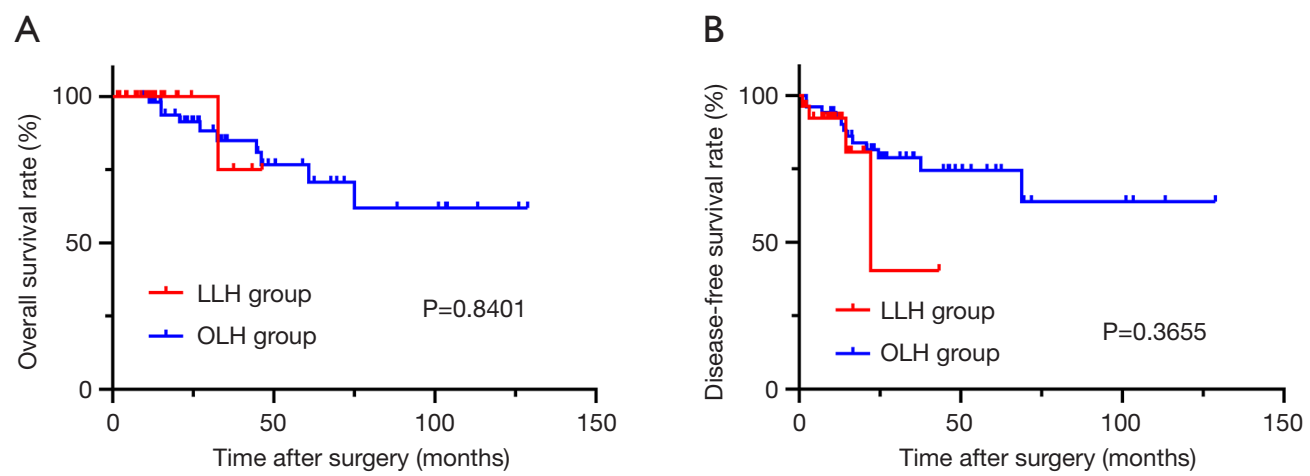

Figure 1 Kaplan-Meier overall survival (OS) (A) and disease-free survival (DFS) (B) curves of HCC patients in LLH and OLH group. Survival difference was analyzed using log-rank test. LLH, laparoscopic left hemihepatectomy; OLH, open left hemihepatectomy.

The successful operation for these patients suggests that the presence of cirrhosis or large tumor may be not a limiting factor or contraindication for LLH. In addition, it is imperative to perform a PSM analysis in this observational study as we found that patients in LLH group tended to have smaller tumor size $(\mathrm{P}=0.001)$ and earlier TNM staging $(\mathrm{P}=0.022)$. This may reflect the preference of surgeons in selecting patients for LLH. As we wished, the different distributions of covariates between LLH and OLH groups, especially like AFP, tumor size, cell differentiation, MVI, and TNM staging, were well balanced after PSM.

There was a tendency of less intraoperative blood loss and lower hepatic portal occlusion rate in patients undergoing LLH in comparison with those in OLH group. Besides, we found that the transfusion rate was not significantly different between the two groups after matching. Similar results were reported in previous studies on anatomic hepatectomy $(17,18,29)$. The control of bleeding is an important part of surgery and thus sometimes hepatic inflow occlusion like Pringle maneuver is necessary during the parenchymal transection. During the operation of LLH, our team generally use the Glissonean pedicle approach of left hepatic artery and portal vein for inflow control, which can reduce the incidence of ischemiareperfusion injury and gastrointestinal congestion. At the same time, ultrasound and intraoperative indocyanine green-fluorescence imaging technique are used for identification of the Glissonean system and margin of liver segments, respectively. For parenchymal transection, ultrasonic dissection and stapler are most commonly used and the middle hepatic vein should be exposed on the cutting plane for better blood control. In case of uncontrollable bleeding, conversion to the open surgery should be performed without hesitation. These experiences 
in LLH help us effectively control intraoperative blood loss. The reduction of bleeding in LLH is believed to be derived from some additional advantages of laparoscopy. For example, the establishment of pneumoperitoneum could lead to a lower central venous pressure $(1,23)$ and the magnification of laparoscopy allows for a more meticulous dissection of liver parenchyma (1). The less application of hepatic portal occlusion in LLH group indicated a decreased risk of liver ischemia-reperfusion injury and dysfunction during the laparoscopic surgery.

Resection margin is an important factor that could influence long-term survival (23). Although it was initially considered that LH would compromise the resection margin due to the lack of palpation (29), the magnification of laparoscopy, the anatomical resection, and the routine application of laparoscopic ultrasound may compensate the deficiency of palpation. In this study, consistent with other researches $(7,24,25,28,29)$, no significant difference in surgical margin was observed between the two groups.

The frequency and severity of postoperative complications are considered as important evaluations of surgical quality. Although the rate of complications (Clavien-Dindo classification II or above) in LLH group was lower than that in OLH group ( $0.0 \%$ versus $13.3 \%)$, no statistical difference was presented in this study. However, another study reported a significantly lower postoperative morbidity of LLH group (17). Different results may be attributed to the small cohorts of patients and variances in surgical operations across teams in different studies.

Finally, we found that patients in LLH group experienced comparable OS and DFS to those in the OLH group. The long-term postoperative prognosis is the major controversy of LH for HCC treatment (11). According to the results of our and other studies $(18,33,34)$, the surgical method appears not to have an obvious influence on oncological outcomes of HCC patients. It may be partially explained by the similar surgical details like resection margin and rate of complications between LLH and OLH groups. Nevertheless, more supportive evidence is warranted to get the final conclusion.

There are some limitations in this study. First, the design of retrospective observation in a single surgical center makes it necessary to conduct prospective, multicenter studies in the future. Nevertheless, the operation of LLH is generally restricted to specific tertiary hospitals, which makes the external validation of our study findings challenging. Second, due to the complicated procedures and a steep learning curve, the number of patients undergoing LLH is limited. The small sample size has an adverse impact on statistical reliability and validity, which may affect the validity of our findings like the incidence of postoperative complications mentioned earlier. Though PSM analysis is performed to reduce selection bias as much as possible, results of study with a large cohort of patients will be more convincing. Third, the follow-up period should be longer.

\section{Conclusions}

In conclusion, this study is valuable in view that research on the application of LLH for HCC patients is relatively lacking. Despite the limited sample size and need for further research, the current study demonstrates the potential benefit of LLH on short-term outcomes of HCC patients without compromising the oncological prognosis. LLH may be a feasible and safe alternative to OLH for selected HCC patients, even in those with cirrhosis and large tumors.

\section{Acknowledgments}

Funding: This work was supported by the Shanghai International Science and Technology Collaboration Program (18410721900), the Shanghai Science and Innovation Action Plan Program (19511121304), the National Natural Science Foundation of China (81472672).

\section{Footnote}

Reporting Checklist: The authors have completed the STROBE reporting checklist. Available at http://dx.doi. org/10.21037/tcr-20-1573

Data Sharing Statement: Available at http://dx.doi. org/10.21037/tcr-20-1573

Conflicts of Interest: All authors have completed the ICMJE uniform disclosure form (available at http://dx.doi. org/10.21037/tcr-20-1573). The authors have no conflicts of interest to declare.

Ethical Statement: The authors are accountable for all aspects of the work in ensuring that questions related to the accuracy or integrity of any part of the work are appropriately investigated and resolved. The study was conducted in accordance with the Declaration of Helsinki (as revised in 2013). A written informed consent for operation was obtained from each patient and this study was approved 
by the Clinical Research Ethics Committee of Zhongshan Hospital, Fudan University (ID: Y2018-115).

Open Access Statement: This is an Open Access article distributed in accordance with the Creative Commons Attribution-NonCommercial-NoDerivs 4.0 International License (CC BY-NC-ND 4.0), which permits the noncommercial replication and distribution of the article with the strict proviso that no changes or edits are made and the original work is properly cited (including links to both the formal publication through the relevant DOI and the license). See: https://creativecommons.org/licenses/by-nc-nd/4.0/.

\section{References}

1. Cheung TT, Dai WC, Tsang SH, et al. Pure Laparoscopic Hepatectomy Versus Open Hepatectomy for Hepatocellular Carcinoma in 110 Patients With Liver Cirrhosis: A Propensity Analysis at a Single Center. Ann Surg 2016;264:612-20.

2. Xiang L, Li J, Chen J, et al. Prospective cohort study of laparoscopic and open hepatectomy for hepatocellular carcinoma. Br J Surg 2016;103:1895-901.

3. Yoon YI, Kim KH, Cho HD, et al. Long-term perioperative outcomes of pure laparoscopic liver resection versus open liver resection for hepatocellular carcinoma: a retrospective study. Surg Endosc 2020;34:796-805.

4. Li W, Han J, Xie G, et al. Laparoscopic versus open mesohepatectomy for patients with centrally located hepatocellular carcinoma: a propensity score matched analysis. Surg Endosc 2019;33:2916-26.

5. Di Sandro S, Bagnardi V, Najjar M, et al. Minor laparoscopic liver resection for Hepatocellular Carcinoma is safer than minor open resection, especially for less compensated cirrhotic patients: Propensity score analysis. Surg Oncol 2018;27:722-9.

6. Xourafas D, Pawlik TM, Cloyd JM. Early Morbidity and Mortality after Minimally Invasive Liver Resection for Hepatocellular Carcinoma: a Propensity-Score Matched Comparison with Open Resection. J Gastrointest Surg 2019;23:1435-42.

7. Untereiner X, Cagniet A, Memeo R, et al. Laparoscopic Hepatectomy Versus Open Hepatectomy for the Management of Hepatocellular Carcinoma: A Comparative Study Using a Propensity Score Matching. World J Surg 2019;43:615-25.

8. Tsai KY, Chen HA, Wang WY, et al. Long-term and short-term surgical outcomes of laparoscopic versus open liver resection for hepatocellular carcinoma: might laparoscopic approach be better in early HCC? Surg Endosc 2019;33:1131-9.

9. Deng ZC, Jiang WZ, Tang XD, et al. Laparoscopic hepatectomy versus open hepatectomy for hepatocellular carcinoma in 157 patients: A case controlled study with propensity score matching at two Chinese centres. Int J Surg 2018;56:203-7.

10. Rhu J, Kim SJ, Choi GS, et al. Laparoscopic Versus Open Right Posterior Sectionectomy for Hepatocellular Carcinoma in a High-Volume Center: A Propensity Score Matched Analysis. World J Surg 2018;42:2930-7.

11. Cai X. Laparoscopic liver resection: the current status and the future. Hepatobiliary Surg Nutr 2018;7:98-104.

12. Bray F, Ferlay J, Soerjomataram I, et al. Global cancer statistics 2018: GLOBOCAN estimates of incidence and mortality worldwide for 36 cancers in 185 countries. CA Cancer J Clin 2018;68:394-424.

13. El-Serag HB, Marrero JA, Rudolph L, et al. Diagnosis and treatment of hepatocellular carcinoma. Gastroenterology 2008;134:1752-63.

14. Zhou C, Chen W, Sun J, et al. Low expression of WW domain-containing oxidoreductase associates with hepatocellular carcinoma aggressiveness and recurrence after curative resection. Cancer Med 2018;7:3031-43.

15. Buell JF, Cherqui D, Geller DA, et al. The international position on laparoscopic liver surgery: The Louisville Statement, 2008. Ann Surg 2009;250:825-30.

16. Komatsu S, Brustia R, Goumard C, et al. Laparoscopic versus open major hepatectomy for hepatocellular carcinoma: a matched pair analysis. Surg Endosc 2016;30:1965-74.

17. Zhang Y, Huang J, Chen XM, et al. A Comparison of Laparoscopic Versus Open Left Hemihepatectomy for Hepatocellular Carcinoma. Surg Laparosc Endosc Percutan Tech 2016;26:146-9.

18. Chen K, Pan Y, Wang YF, et al. Laparoscopic Right Hepatectomy for Hepatocellular Carcinoma: A Propensity Score Matching Analysis of Outcomes Compared with Conventional Open Surgery. J Laparoendosc Adv Surg Tech A 2019;29:503-12.

19. Katayama H, Kurokawa Y, Nakamura K, et al. Extended Clavien-Dindo classification of surgical complications: Japan Clinical Oncology Group postoperative complications criteria. Surg Today 2016;46:668-85.

20. Austin PC. An Introduction to Propensity Score Methods for Reducing the Effects of Confounding in Observational Studies. Multivariate Behav Res 2011;46:399-424. 
21. Reich H, McGlynn F, DeCaprio J, et al. Laparoscopic excision of benign liver lesions. Obstet Gynecol 1991;78:956-8.

22. Aldrighetti L, Guzzetti E, Pulitano C, et al. Case-matched analysis of totally laparoscopic versus open liver resection for HCC: short and middle term results. J Surg Oncol 2010;102:82-6.

23. Cheung TT, Poon RT, Yuen WK, et al. Long-term survival analysis of pure laparoscopic versus open hepatectomy for hepatocellular carcinoma in patients with cirrhosis: a single-center experience. Ann Surg 2013;257:506-11.

24. Aldrighetti L, Pulitano C, Catena M, et al. A prospective evaluation of laparoscopic versus open left lateral hepatic sectionectomy. J Gastrointest Surg 2008;12:457-62.

25. Garbarino GM, Marchese U, Tobome R, et al. Laparoscopic versus open unisegmentectomy in two specialized centers. Feasibility and short-term results. HPB (Oxford) 2020;22:750-6.

26. Fu XT, Tang Z, Shi YH, et al. Laparoscopic Versus Open Left Lateral Segmentectomy for Large Hepatocellular Carcinoma: A Propensity Score-Matched Analysis. Surg Laparosc Endosc Percutan Tech 2019;29:513-9.

27. Kim JM, Kwon CHD, Yoo H, et al. Which approach is preferred in left hepatocellular carcinoma? Laparoscopic versus open hepatectomy using propensity score matching. BMC Cancer 2018;18:668.

Cite this article as: Yi Y, Weng J, Zhou C, Liu G, Ren N. Laparoscopic versus open left hemihepatectomy for hepatocellular carcinoma: a propensity score matching analysis. Transl Cancer Res 2020;9(9):5484-5492. doi: 10.21037/tcr-20-1573
28. Truant S, Bouras AF, Hebbar M, et al. Laparoscopic resection vs. open liver resection for peripheral hepatocellular carcinoma in patients with chronic liver disease: a case-matched study. Surg Endosc 2011;25:3668-77.

29. Tranchart H, Di Giuro G, Lainas P, et al. Laparoscopic resection for hepatocellular carcinoma: a matched-pair comparative study. Surg Endosc 2010;24:1170-6.

30. Dagher I, O'Rourke N, Geller DA, et al. Laparoscopic major hepatectomy: an evolution in standard of care. Ann Surg 2009;250:856-60.

31. Kluger MD, Vigano L, Barroso R, et al. The learning curve in laparoscopic major liver resection. J Hepatobiliary Pancreat Sci 2013;20:131-6.

32. Shen Y, Zhou C, Zhu G, et al. Liver Stiffness Assessed by Shear Wave Elastography Predicts Postoperative Liver Failure in Patients with Hepatocellular Carcinoma. J Gastrointest Surg 2017;21:1471-9.

33. Yoon YI, Kim KH, Kang SH, et al. Pure Laparoscopic Versus Open Right Hepatectomy for Hepatocellular Carcinoma in Patients With Cirrhosis: A Propensity Score Matched Analysis. Ann Surg 2017;265:856-63.

34. Wang ZY, Chen QL, Sun LL, et al. Laparoscopic versus open major liver resection for hepatocellular carcinoma: systematic review and meta-analysis of comparative cohort studies. BMC Cancer 2019;19:1047. 